\title{
Hipoglicemia y Recambio
}

\author{
Drs. J.P. Beca*, R. Filippa* y C. Román*
}

Desde que Hartman y Jaudon, en $1937^{\circ}$, llaman la atención sobre la posible asociación de niveles sanguíneos bajos de glucosa con la aparición de ciertos síntomas neurológicos en recién nacidos, numerosos autores han destacado la importancia de la hipoglicemia como causa posible de secuelas neurológicas ${ }^{2-3-11-12-13-14}$ 16. La aceptación general del riesgo de la hipoglicemia justifica el esfuerzo que se realiza actualmente para prevenir esta condición para lo cual es esencial, junto a los esquemas de alimentación precoz y a los controles de laboratorio, identificar lo más precisamente posible a los recién nacidos en mayor riesgo. Se considera mayormente expuestos al riesgo de hipoglicemia a los recién nacidos de pretérmino, a los desnutridos fetales, a los hijos de madre diabética, a los casos de hipoxia neonatal severa y de dificultad respiratoria, a los recién nacidos con enfermedad hemolitica por incompatibilidad Rh y a los sometidos a exsangúneo-transfusión independientemente de la causa de ictericia.

La exsanguíneo-transfusión realizada con sangre heparinizada puede potenciar hipoglicemia debido a la baja concentración de glucosa en la sangre del dador ${ }^{18-19}$, especialmente en casos de enfermedad hemolítica por incompatibilidad $R h$. Se ha demostrado, también, que en los recambios realizados con sangre conservada en $\mathrm{ACD}$ se produce una elevación inicial de la glicemia posrecambio, seguida de un descenso progresivo que lleva en algunos casos a valores anormalmente bajos a las 2 y 3 horas después. Se ha comprobado en estos casos un hiperinsulinismo transitorio que explica este efecto hipoglicemiante tardío del recambio efectuado con sangre citratada ${ }^{1-4-18-19}$.

En el Hospital L. Calvo Mackenna los recam-

* Cencro de Prematuros, Hospital Luis Calvo Mackenna. bios sanguineos se efectúan desde 1965, utilizando sangre conservada en ACD y posteriormente adicionada de 1.500 unidades de heparina $y$ de $5 \mathrm{ml}$ de gluconato de calcio $10 \%$ por cada $500 \mathrm{ml}$ de sangre. Este método ha significado diversas ventajas que han sido publicadas anteriormente ${ }^{9}$. Sin embargo, al quedar la duda sobre el comportamiento de la glicemia después de los recambios efectuados con esta técnica se diseñó un estudio prospectivo determinando glicemias seriadas después de los recambios.

\section{MATERIAL Y METODOS}

Se estudió prospectivamente a 19 recién nacidos hospitalizados en el Centro de Prematuros del Hospital L. Calvo Mackenna, en quienes se practicaron 20 recambios por causas diversas.

Trece de los 19 recién nacidos no recibieron soluciones endovenosas antes ni durante los recambios y constituyeron el grupo principal de estudio. Los recambios en estos nin̄os se indicaron por jctericia por defecto de conjugación en 6 casos, por enfermedad hemolitica por incompatibilidad ABO en 4 casos, y por enfermedad hemolítica por incompatibilidad $R h$ en 3 recién nacidos. La edad gestacional promedio fue $38,6 \pm 2,1$ semanas y el peso de nacimiento promedio $3.043 \pm 567 \mathrm{grs}$.

En un segundo grupo, compuesto por 6 recién nacidos, se indicó el recambio mientras recibían soluciones glucosadas endovenosas debido a su bajo peso, por ser pequeños para su edad gestacional, o por sospecha clinica de hipoglicemia. Uno de estos niños fue recambiado dos veces. En ninguno de ellos se suspendió la fleboclisis durante el recambio y siempre se continuó con el mismo goteo/minuto una vez finalizado éste.

La alimentación de todos los niños estudiados 
fue con una mezcla de Eledón diluido al $7 \%$ y adicionado de $4 \%$ de maltosa-dextrina, $3 \%$ de sacarosa y $2,5 \%$ de aceite vegetal. La alimentación oral se continuó en los horarios habituales tanto antes como después del recambio, suspendiéndose sólo cuando el recambio se efectuó en la misma hora en que se debiera haber administrado la alimentación.

Los recambios se efectuaron entre las 2 horas y los 7 dias de edad. Todos ellos se realizaron por vena umbilical con 2 a 2.5 volumenes de sangre conservada en $70 \mathrm{ml}$ de C.PD (ácido cítrico L.S.P. $0,327 \%$, citrato de sodio U.S.P. $2,63 \mathrm{~g} \%$, losfato sódico monobásico U.S.P. $0,222 \mathrm{~g} \%$, y dextrosa U.S.P. $2,55 \mathrm{~g} \%$ ) y adicionada de $1.500 \mathrm{U}$ de heparina y de $5 \mathrm{ml}$ de gluconato de calcio $10 \%$ por cada $500 \mathrm{ml}$. La glicemia determinada en la sangre del dador tuvo un valor promedio de $388 \pm 13.4 \mathrm{mg} \%$.

A los niños de ambos grupos se determinó glicemia inmediatamente antes, al finalizar, y una, dos y tres horas después del recambio.

Las glicemias se determinaron empleando el método de Somogy-Nelson en muestras de $0,2 \mathrm{ml}$ de sangre capilar ${ }^{20}$.

\section{RESULTADOS}

Los resultados de las determinaciones de glicemia efectuadas a los 13 niños recambiados que no recibieron fleboclisis aparecen en la tabla 1. Aparecen en ella los diagnósticos causales de la ictericia, y los valores de glicemia encontrados antes del recambio, al finalizar el recambio. y una, dos y tres horas posrecambio. Ninguno de los niños estudiados presentó hipoglicemia hasta tres horas después del recambio, siendo el valor individua! más bajo la glicemia de $41 \mathrm{mg} \%$ que presentó el caso 5 en la muestra tomada dos horas posrecambio. T'ampoco hubo un valòr excesivamente elevado de glicemia. siendo la glicemia más alta $182 \mathrm{mg} \%$ al terminar la exsanguíneo-transfusión en el caso 3. Los valores promedios aparecen en la tabla 1 y en el grálico, señalándose en ambos la variación de una desviación estándar.

Tabla 1

INDICACION DEL RECAMB]O Y VALORES DE GLJCEMIA (my\%) EN 13 NIÑOS QUE NO RECIBIERON SOLUCION GLUCOSADA ENDOVENOSA

\begin{tabular}{|c|c|c|c|c|c|c|}
\hline \multirow[b]{2}{*}{ Caso $\Lambda^{\circ}$} & \multirow{2}{*}{ Indicación } & \multicolumn{5}{|c|}{ Gitcemio (mg\%) } \\
\hline & & Prerrec. & Postrec. & $-I h r$ & $2 h r$ & $3 h r$ \\
\hline 1 & $\mathrm{ABO}$ & 66 & 132 & 129 & 62 & 51 \\
\hline 2 & def oonj. & 122 & 125 & 68 & 92 & 100 \\
\hline 3 & def. conj. & 101 & 182 & 101 & 150 & 110 \\
\hline 4 & def. $\infty$ j. & 82 & 159 & 86 & 90 & 70 \\
\hline 5 & incomp. Rh & 47 & 112 & 56 & 41 & 42 \\
\hline 6 & incomp- Rh & 47 & 109 & 82 & 93 & 66 \\
\hline 7 & def. $\infty$ j. & 66 & 140 & 114 & 97 & 68 \\
\hline 8 & def. conj. & 47 & 132 & 117 & 76 & 76 \\
\hline 9 & $\mathrm{ABO}$ & 68 & 80 & 98 & 82 & 62 \\
\hline 10 & $\mathrm{ABO}$ & 66 & 121 & 78 & 86 & 77 \\
\hline 11 & incomp. Rh. & 50 & 113 & 96 & 89 & 86 \\
\hline 12 & ABO & 74 & 121 & 98 & 82 & 72 \\
\hline 13 & def. conj. & 84 & 104 & 74 & 65 & 90 \\
\hline \multicolumn{2}{|c|}{ PROMEDIO \pm 1 D.S. } & $31 \pm 20$ & $125 \pm 25$ & $92 \pm 20$ & $85 \pm 24$ & $75 \pm 18$ \\
\hline
\end{tabular}

Los resultados de las glicemias determinadas después de los 7 recambios efectuados a niños que recibieron fleboclisis de solución glucosada $10 \%$ durante el procedimiento aparecen en la tabla 2. No se calcularon promedios en este grupo dada la gran dispersión de los re- sultados. Debe destacarse que en este grupo hubo 3 de $\operatorname{los} 7$ casos (casos 1,3 y 6 ) con valores de glicemia sobre $300 \mathrm{mg} \%$. Ninguno de los niños de este grupo presentó hipoglicemia posrecambio. Uno de ellos (caso 5-a) tenía una hipoglicemia asintomática al iniciarse la 


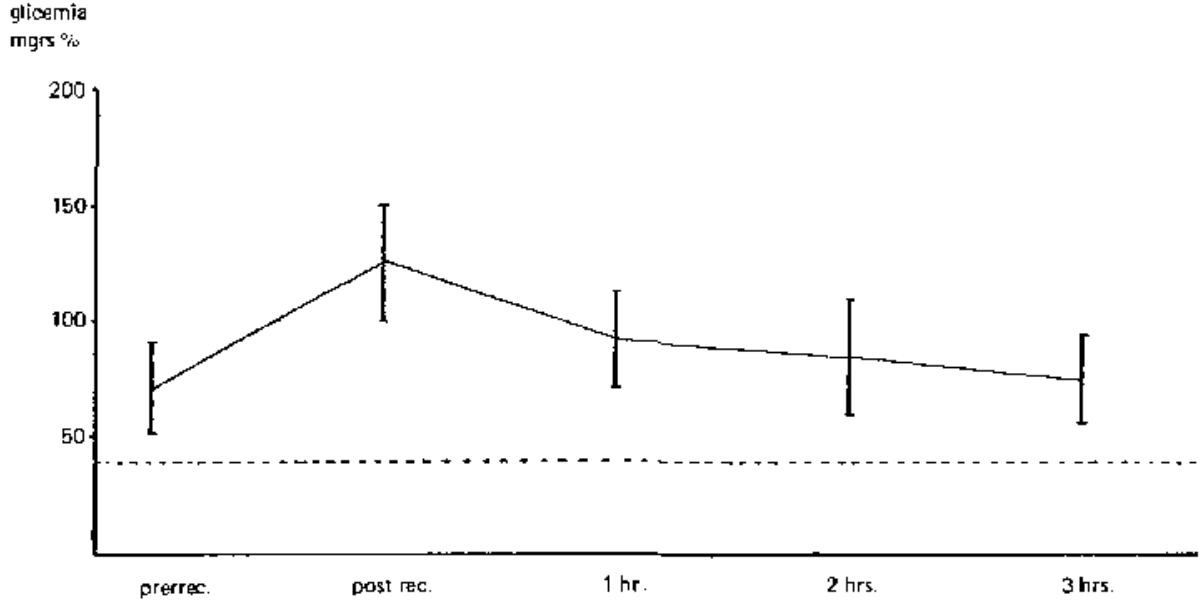

Tabla 2

INLICIACION Y VALORES DE GLICEMIA (mg\%) EN 7 RECAMBIOS EFECTTUADOS A 6 RECILN NACIDOS QUE RECIBIERON SOLUCION GLUCOSADA DURANTE EL PROCEIJMIF.NTO

\begin{tabular}{|c|c|c|c|c|c|c|}
\hline \multirow[b]{2}{*}{$\operatorname{Casos} \lambda^{\circ}$} & \multirow[b]{2}{*}{ Indicación } & \multicolumn{5}{|c|}{ Ghecmia (mg\%) } \\
\hline & & Premex. & Postres. & $t h r$ & $2 \mathrm{hr}$ & $3 \mathrm{hr}$ \\
\hline 1 & del. canj. & 214 & 442 & 302 & 178 & 171 \\
\hline 2 & incomp. $R h$ & 85 & 100 & $9 \mathrm{~B}$ & 56 & 48 \\
\hline 3 & def. conj. & 79 & 390 & 370 & 305 & 182 \\
\hline 4 & $A B O$ & 53 & 44 & $\cdot 154$ & $1: 2$ & -. \\
\hline $5 a$ & incomp. Rh & 15 & 181 & 105 & 96 & $7 B$ \\
\hline $5 b$ & incomp. $\mathrm{Rh}$ & 82 & 137 & 252 & 102 & 108 \\
\hline 0 & def. conj- & 69 & 540 & 540 & $\$ 15$ & $4 \%$ \\
\hline
\end{tabular}

exsangúnen-transfusión, corrigiéndose este valor á finalizar el procedimiento y permaneciendo con cifras normales hasta tres horas después.

\section{DISCUSION}

Los resultados de esta serie muestran que ninguno de los niños estudiados presentó hipoglicemia posrecambio, lo cual contrasta con los resultados obtenidos por Schiff y cols. ${ }^{19}$. quienes encontraron después de recambios efectuados con sangre conservada en ACD. hipoglicemia en 5 de los 10 niños previamente normoglicémicos, y en el total de los 5 niños previamente hipoglicemicos. Schiff y cols. demostraron, al igual que Cser y Milner", un hiperinsulinismo transitorio posrecambio cuando éste se efectuó con sangre conservada en ACD. El hiperinsulinismo puede explicar esta rendencia a la hipoglicemia después de los re- cambios efectuados con esta técnica. especialmente en los casos de enfermedad hemolítica por incompatibilidad $\mathrm{Rh}^{15}{ }^{17}$. Los casos de enlermedad hemolitica por $\mathbf{R h}$ de l.s presente serie tampoco mostraron tendencia is la hipoglicemia posrecambio.

Schiff $y$ cols. $^{\text {lo }}$ y Cser y Milner demostraron que en los recambios electuados con sangre heparinizada no se modifican significativamente los niveles de glucosa ni de insulina, ni durante ni después del mismo. Sin embargo se produce en estos casos una elevación importante de los niveles plasmáticos de ácidos grasos libres, lo cual puede significar riesgo por cuanto los ácidos grasos libres compiten con la Bilirrubina no conjugada en su unión a albúmina. Pero el aumento de ácidos grasos libres plasmáticos inhibe la captación y oxidación de glucosa en tejidos periféricos, especialmente en 
los músculos, lo cual representa un mecanismo a ciclo regulador de los niveles sanguíneos de glucosa ${ }^{10-1 b}$. Habiéndose realizado los recambios de esta serie con sangre conservada en ACD y además heparinizada, puede suponerse que se produjo en estos niños un hiperinsulinismo secundario a los niveles elevados de glucosa en la sangre del dador, junto a un aumento de la concentración de ácidos grasos libres en el plasma por la activación de la lipolisis que produce la heparinat. Se podria entonces especular que el efecto hipoglicemiante de los recambios efectuados con sangre conservada en ACD se vería disminuido por el aumento de ácidos grasos libres producido al heparinizar la sangre del dador.

Los niños que fueron recambiados mientras recibían suero glucosado endovenoso presentaron niveles muy variados de glicemta posrecambio. Ninguno de ellos presentó hipoglicemia, y uno de ellos mejoró de una hipoglicemia asintomática preexistente. Sin embargo hubo glicemias superiores a $300 \mathrm{mg} \%$ después de 3 de $\operatorname{los} 7$ recambios efectuados en estos 6 nin̄os. Dweck y Cassady ${ }^{8}$ han resaltado el riesgo de la hiperglicema como posible factor causal de hemorragia intracraneana en recién nacidos, por lo que creemos que esta condición debe ser evitada. Se pudo comprobar que durante los recambios se produjo un aumento involuntario de la velocidad de infusión del suero endovenoso explicable por los cambios de presión venosa. Creemos que los casos de hiperglicemia posrecambio pueden explicarse por dichas variaciones en las velocidades de infusión de las fleboclisis durante los recambios.

Los resultados presentados permiten concluir que, cuando los recambios se efectúan con sangre conservada en ACD y posteriormente heparinizada, no exjste un riesgo importante de hipoglicemia posrecambio, en especial si se alimenta precozmente a los niños después del procedimiento. Por otra parte, cuando los niños a quienes se practica recambio están recibiendo soluciones glucosadas endovenosas parece recomendable suspenderlas como una manera de evitar la hiperglicemia.

\section{RESUMEN}

Sc estudió prospectivamente 19 recién nacidos en quienes se efectuó exsanguinco-transfusión por diversas causas. El recambio se pracicó empleando sangre conservada en C.P.D. y adicionada de $1.500 \mathrm{U}$ de heparina y de $5 \mathrm{ml} \mathrm{de} \mathrm{glu-}$ conato de calcio $10 \%$. La glicemia de la sangre del dador fue en promedio $388 \pm 13.4 \mathrm{mg} \%$. A todos los niños se practicó glicemia inmediatamente antes y al finalizar el procedimiento, así como una, dos y tres horas despues.

Tirece niños no recibieron soluciones parenıerales, y ninguno de ellos presentó valores de glicemia bajo $40 \mathrm{mg} \%$ ni sobre $200 \mathrm{mg} \%$ durante el periodo de estudio. Seis pacientes fueron recambiados mientras recibian solución glucosada $10 \%$ intravenosa. Ninguno de ellos presentó hipoglicemia posrecambio, pero despues de 3 de los 7 recambios efectuados en este grupo, se encontró valores sobre $300 \mathrm{mg} \%$

Se concluye que cuando la exsang uíneotransfusión se efectúa con sangre conservada en C.P.D. y adicionada de heparina se reduce el ritsgo de hipoglicemia posrecambio. En aquellos monos que estan recibiendo solución glucosada se recomienda suspenderla antes de iniciar el procedimiento para evitar una posible hiperglicemia.

\section{SUMMARY}

A group of 19 newburn infanis who were blood-exchanged was studied. Exchange transfusions were performed using C.P.D. blood to which $1.500 \mathrm{U}$ of Heparin and $5 \mathrm{ml}$ of calcium gluconate $/ 500 \mathrm{ml}$ were added. Mean blood glucose in donor's blood was $388 \pm 13,4 \mathrm{mg} \%$. Glycemia was determined in all patients inmediately before the exchange procedure and at its completion as well as al one, two and three hours post-exchange.

Thirteen infants did not receive parenteral solutions and nowe of them had bloxd glucose Jrvels below $40 \mathrm{mg} \%$ or over $200 \mathrm{mg} \%$ during the study period. Six patients were exchanged while receiving $10 \%$ glucose solution intraveonously. None of them had post-exchange hypoglycerria; but biood gilucose values greater than $300 \mathrm{mg} \%$ were found after 3 of the 7 exchangetransfusions performed in this group.

Authors conclude that if the donors blood has C.P.D and Heparin added to it the risk of post-exchange hypoglycerria is reduced. In those patients who are receiving parenteral glucose solutions, these should be discontinued before initiating the procedure in onder to avoid possible post-exchange hiperglycemia.

\section{REFERENCIAS}

I Barret C.T. Otiver T.K. N. Engl. J. Med., 278: 1260. 1968 .

- Comblath M., Juasin G. a al. Pediat. Clin N. Amer., 13:005, 1966.

3 Comblath, M.; Ozand, P.T. Neonatal Hypogyycemia, Abstracts XIV Intemational Cangress of Pediatrics, I: 13,1974

- Cser, A.; Milnet, R.D.G. Arch. Dis, Child., 49:940, 1974

- Dnscoll, S.G.; Sieinke. J. Pediatrics, 39:448, 1967.

- Dweek, H.S.; Cassady, G. Pediatrics, 53:189, 1974

- From, G.L.A.; Driscoll, S.G.; Steinke, J. Pediatrics, 44:549, 1969. 
- Hortman, A.F.; Jaudon, J.C. Hypoglycemia. J. Pediat., $11: 1,193$ ?

- Howard, J.E.; Hudobro, J.C.; Allende, $\mathrm{N}$; Sánchez, M. Rev. Ch. Ped., 36:642, 1965.

10 Hull, D. The function and development of adipose issue, Scientific Foundations of Yediatrics, J. Davis \& J. Dobbing, V. Heinemann Medical Books Led London, 1974.

"Lubchenco, L,O.; Bard, H. Pediatries, 47:831, 19 ? 1.

12 Neligan, C. Idiopathic bypoglycemia in the newborn, Recent Advances in Pediatrics, 3* Ed., London. 1965.

1s Pagliara, A.S; Kart, I.E.; Haymont, M.; Kipnis, D.M. J. Pediat., 82:365, 1973.

14 Pildes, R; Forbes, A.E; Otonnur, S.M.; Combloth, M. J. Pediat. JC: 76, 1967 .

15 Rondte, P.J.; Garland, P.B.; Hales, C.S.: Veritholme, E.A. Lancet, $1: 785,1963$. 1e Román, $C_{\text {: }}$ Beca, J.f.; Fithppa, R. Hipoglicembi en recién nacidos de poco peso. Rev. (h. Ped (en prensa)

it Schuff. D.; Lozwy, C: Pediat., Res. 4:280, 1970.

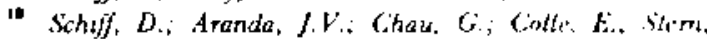
I. J. Pediat.. 78:603, 1971.

1. Scheff, D.; A randa, J.V; Colle, E.; Stem. I. J. Pediat1.. $79: 589,197 \mathrm{t}$

30 Simogy, M. J. Biot. Chem , 161):69, 1945.

\section{AGRAJEC:IMIEN'IOS:}

Las autores agradecen a C;. Parde por su teahajo de laboira. rorio $y$ a las enfermeras y auxiliares del Centro de Premaluros por el eficiente $y$ ahnegado cuidado de los niñus a sn (argo 